\title{
Phenotypic and genetic characterization of resistance in Arabidopsis thaliana to the oomycete pathogen Phytophthora parasitica
}

Yuling Meng1,2, Yihua Huang 1,2, Qinhu Wang ${ }^{2,3}$, Qujiang Wen ${ }^{1,2}$, Jinbu Jia ${ }^{1,2}$, Qiang Zhang 1,2, Guiyan Huang ${ }^{2,3}$, Junli Quan ${ }^{1,2}$ and Weixing Shan ${ }^{1,2 *}$

${ }^{1}$ College of Plant Protection, Northwest A\&F University, Yangling, China, ${ }^{2}$ State Key Laboratory of Crop Stress Biology for Arid Areas, College of Plant Protection, Northwest A\&F University, Yangling, China, ${ }^{3}$ College of Life Sciences, Northwest A\&F University, Yangling, China

\section{OPEN ACCESS}

Edited by:

Pietro Daniele Spanu, Imperial College London, UK

Reviewed by:

Walter Gassmann, University of Missouri, USA

Mahmut Tör,

University of Worcester, UK Sebastian Schornack, University of Cambridge, UK

*Correspondence: Weixing Shan, State Key Laboratory of Crop Stress Biology for Arid Areas, College of Plant Protection, Northwest A\&F University, 3 Taicheng Road, Yangling,

Shaanxi 712100, China wxshan@nwafu.edu.cn

Specialty section: This article was submitted to Plant-Microbe Interaction, a section of the journal Frontiers in Plant Science

Received: 03 February 2015 Accepted: 12 May 2015 Published: 27 May 2015

Citation:

Meng $Y$, Huang $Y$, Wang $Q$, Wen $Q$,

Jia J, Zhang Q, Huang G, Quan J and Shan W (2015) Phenotypic and genetic characterization of resistance in Arabidopsis thaliana to the oomycete pathogen Phytophthora parasitica. Front. Plant Sci. 6:378. doi: $10.3389 /$ fpls.2015.00378
The interaction between Arabidopsis thaliana and the oomycete pathogen Phytophthora parasitica emerges as a model for exploring the molecular basis and evolution of recognition and host defense. Phenotypic variation and genetic analysis is essential to dissect the underlying mechanisms in plant-oomycete interaction. In this study, the reaction phenotypes of $28 A$. thaliana accessions to $P$. parasitica strain Pp016 were examined using detached leaf infection assay. The results showed the presence of four distinct groups based on host response and disease development. Of all the accessions examined, Zurich $(Z \mathrm{u}-1)$ is highly resistant to $P$. parasitica. Microscopic characterization showed that rapid and severe hypersensitive response at the primary infection epidermal cells is associated with disease resistance. Furthermore, Zu-1 is resistant to a set of 20 diverse $P$. parasitica strains, which were collected from different host plants and exhibited differential specificities on a set of tobacco cultivars. However, $\mathrm{Zu}-1$ is susceptible to $P$. parasitica when the root is inoculated, suggesting differential expression of associated resistance genes in the root and foliar tissues. Genetic analysis by crossing Zu-1 and the susceptible accession Landsberg (Ler) showed that the resistance in $\mathrm{Zu}-1$ to $P$. parasitica is semi-dominant, as shown by infection assays of $F_{1}$ progenies, and is likely conferred by a single locus, defined as RPPA $1^{Z u-1}$ (for Resistance to $P$. parasitica 1), as shown by analysis of $F_{2}$ segregating populations. By employing specific-locus amplified fragment sequencing (SLAF-seq) strategy to identify molecular markers potentially linked to the locus, the strongest associated region was determined to be located between 7.1 and $11.2 \mathrm{Mb}$ in chromosome IV. The future cloning of $R P P A 1^{Z u-1}$ locus will facilitate improved understanding of plant broad-spectrum disease resistance to oomycete pathogens.

\section{Keywords: Arabidopsis thaliana, Phytophthora parasitica, resistance, Zu-1, genetic analysis}

\section{Introduction}

Oomycetes represent a group of eukaryotic microorganisms related to diatoms and brown algae, causing many destructive diseases to plants and animals (Beakes et al., 2012). Among the group, 
Phytophthora is the best-studied genus that includes over 100 species, which are divided into 10 clades (Kroon et al., 2012). Phytophthora parasitica Dastur (syn. P. nicotianae Breda de Haan) is classified in the Phytophthora clade 1 and its closest relatives include $P$. infestans (Blair et al., 2008; Kroon et al., 2012). Unlike the well-studied species $P$. infestans, which is a foliage pathogen and only infected few plants, $P$. parasitica is a typical root pathogen with a broad-range of host plants, being capable of infecting over 72 plant genera (Meng et al., 2014). The two species vary in genome sizes, being 82 and $240 \mathrm{Mb}$ for $P$. parasitica and $P$. infestans, respectively (Judelson, 2012). However, nearly half of Phytophthora species are mostly pathogenic on roots, and about $30 \%$ species being pathogens of multiple host plants (Kroon et al., 2012). Therefore, P. parasitica provides an opportunity for their role in understanding plant recognition and infection, and their broad host ranges. Moreover, while being a natural pathogen of tobacco species, $P$. parasitica is capable of infecting the model plant species Arabidopsis thaliana, which allows accelerated understanding of Phytophthora pathogenesis and plant susceptibility (Attard et al., 2010; Wang et al., 2011).

During co-evolution of plant host and the pathogen, plants have developed sophisticated recognition systems, especially the effector-triggered immunity (ETI; Jones and Dangl, 2006). The avirulence $(A v r)$ genes from pathogen perceived directly or indirectly by the matching resistance $(R)$ genes following the gene-for-gene model, which leads to a rapid and enhanced defense response in the host plant, often including hypersensitive response (HR; Ali and Bakkeren, 2011). $R$ gene-mediated recognition of pathogen effectors activates a series of defense signaling cascades. So far, numerous $R$ genes have been cloned from many plant species (Liu et al., 2007). The largest class of known $\mathrm{R}$ proteins includes a nucleotidebinding site and leucine-rich repeat domains (NBS-LRR proteins; Joshi and Nayak, 2013). A number of $R$ genes have been cloned from the model species, A. thaliana, and have also been used extensively for answering fundamental questions in molecular plant-microbe interactions, including bacterial, viral, fungal, and oomycete pathogens (Nishimura and Dangl, 2010).

The established interactions between oomycetes and the model plant $A$. thaliana represent an important contribution to the understanding of the oomycete pathogenicity mechanisms (Bozkurt et al., 2012). There have been several examples to use Arabidopsis to investigate plant-oomycete pathogen interactions (Koch and Slusarenko, 1990; Holub et al., 1995; Roetschi et al., 2001; Robinson and Cahill, 2003; Daniel and Guest, 2006; Attard et al., 2010; Schlaeppi et al., 2010; Wang et al., 2011, 2013), especially after the whole genome sequence of Arabidopsis was announced (Arabidopsis Genome Initiative [AGI], 2000). Among the pathosystems established, the best studied model, downy mildew, exhibits extensive variation with A. thaliana, which provides a rich resource for identification of at least 27 RPP genes (Coates and Beynon, 2010), from which several genes have been cloned using map-based cloning. In contrast, little is known about the Avr genes, with only four cloned, including ATR13 (Allen et al., 2004), ATR1 (Rehmany et al.,
2005), ATR5 (Bailey et al., 2011), and ATR39-1 (Goritschnig et al., 2012). In addition, several functional resistance genes from potato and soybean, conferring resistance to $P$. infestans and $P$. sojae, respectively, have also been cloned. Most of the cloned $R$ genes against oomycete pathogens belong to the NBS-LRR class of plant resistance genes (Gururani et al., 2012). More identified $R$ genes and corresponding Avr genes have provided major insights into the mechanism of plantoomycete interactions (Stassen and Van den Ackerveken, 2011).

However, these studies have been limited to race-specific resistance genes. The race-specific $R$ genes are usually difficult to provide long-lived resistance in the field, because the encoded resistance is based on the recognition of corresponding Avr genes. For example, all of the $11 R$ genes originated from Solanum demissum have lost resistance to P. infestans (Song et al., 2003). Broad-spectrum disease resistance, which refers to resistance against different pathogen species or the majority of races of one species, is desirable (Kou and Wang, 2010). However, whether a broad-spectrum resistance gene is durable is still debatable. More than 100 disease-resistance genes have been cloned from different plant species (Liu et al., 2007), and in some cases, several $R$ genes confer broad-spectrum resistance. For broad-spectrum disease resistance, the first type is the resistance to two or more different pathogens, and the second one is defined as the resistance to the majority races of the same pathogen. A good example of the first type is the Arabidopsis $R$ gene $R P W 8$, which confers resistance to two different powdery mildew fungal pathogens, Erysiphe cruciferarum UEA1 and E. cichoracearum UCSC1 (Xiao et al., 2001). $R$ genes of the second type have also been identified (Wang et al., 1996; Büschges et al., 1997). And also, the cloned resistance gene $R B$ from $S$. bulbocastanum enables potato highly resistant to all known races of $P$. infestans (Song et al., 2003), a destructive oomycete pathogen. WRR4 encodes a TIR-NB-LRR (Toll-like/interleukin-1 receptor-nucleotide binding-leucine-rich repeat) protein, confers a dominant, broad-spectrum white rust resistance in Arabidopsis accession Columbia to at least four races of Albugo candida, and requires expression of the lipaselike defense regulator, EDS1 (enhanced disease susceptibility 1; Borhan et al., 2008, 2010). However, the mechanisms of broad-spectrum disease resistance are different. For example, the mlo resistance is a result of the recessive mutations in the barley Mlo locus (Büschges et al., 1997), while in some cases, the durability of $R$ genes lie in the fitness cost in pathogen evolution to overcome the resistance (Vera Cruz et al., 2000). Bs2-mediated broad-spectrum disease resistance is achieved by recognition of avrBs2, an avirulence gene important in the fitness of Xanthomonas campestris pv. vesicatoria and highly conserved among other $X$. campestris pathovars (Tai et al., 1999). In the case of rice bacterial blight, the disease severity was high in 3 years on the $X a 4$ and $X a 10$ genotypes, but not on the $\mathrm{Xa7}$, as the mutation of $a v r X a 7$ was responsible for both the loss of avirulence function and reduced aggressiveness to rice (Vera Cruz et al., 2000). These results suggested that durability of $R$ genes could be predicted according to the fitness or virulence contribution of corresponding avirulence genes. 
In agricultural ecosystem, genetic resistance is the most efficient form of protection against pathogens. $R$ genes have been successfully used in crop improvement programs in the past and are being continuously exploited. Black shank, caused by $P$. parasitica, which is a destructive disease of tobacco worldwide, damages roots, stems, and leaves at any stages of tobacco growth. There are limited known sources of resistance against the pathogen. For example, in the early stage, the only available source of resistance was derived from Fla 301, which exhibited polygenic resistance. Dominant and monogenic resistance from Nicotiana plumbaginifolia Viv (Php) and $N$. longiflora Cav $(P h l)$ was successfully incorporated into burley and flue-cured tobacco cultivars, respectively (Gutiérrez and Mila, 2007; Antonopoulos et al., 2010). However, cultivars with known resistance resources have failed to provide sufficient control against the pathogen in the field. Thus, there is a need to find new resistance resources. Apart from this, it is highly desirable to understand the plant-pathogen interaction in order to develop novel disease-control strategies and improve disease-control measures.

Little is known about the host specificities in $P$. parasitica although its interaction with tobacco plants follows a genefor-gene theory (Perrone et al., 2000) and with A. thaliana shows natural variation in host specificity (Wang et al., 2011). In this study, we used detached leaf inoculation method to investigate phenotypic and genetic interaction between A. thaliana and P. parasitica. Based on careful assessment of variation in resistance phenotypes, we describe in this paper details of four interaction phenotypes. The phenotypic variations in A. thaliana to $P$. parasitica provide useful resources for better understanding of the interaction between Phytophthora and the host plants. We also characterized the resistance of accession Zurich $(\mathrm{Zu}-1)$ to $P$. parasitica strain Pp016. Genetic analysis using segregating populations derived from a cross between the resistant accession $\mathrm{Zu}-1$ and the hypersusceptible accession Landsberg (Ler) show that the resistance is conditioned by a single semi-dominant locus designated $R P P A 1^{Z u-1}$ (for Resistance to $P$. parasitica 1). Since $R P P A 1^{Z u-1}$ confers resistance to diverse $P$. parasitica strains, its future cloning and analysis will facilitate improved understanding of broad-spectrum disease resistance in plants to oomycete pathogens.

\section{Materials and Methods}

\section{Phytophthora parasitica Culture Conditions and Pathogenicity Assays}

The $P$. parasitica strains were routinely cultured on $5 \%(\mathrm{v} / \mathrm{v})$ cleared carrot juice agar (CA) medium supplemented with $0.002 \%(\mathrm{w} / \mathrm{v}) \beta$-sitosterol and $0.01 \%(\mathrm{w} / \mathrm{v}) \mathrm{CaCO}_{3}$ in the dark at $23^{\circ} \mathrm{C}$. The $P$. parasitica zoospore preparation and pathogenicity assays were as described (Wang et al., 2011). For assessing the phenotype of Arabidopsis accessions to $P$. parasitica, at least ten plants were selected (two leaves from each plant) for the pathogenicity assays. The determination of a whole accession as a specific phenotype is based on the infection of leaves of majority plants (more than 70-80\%). All experiments were repeated three times.

\section{Arabidopsis thaliana Growing Conditions}

The preservation of seeds followed a standard protocol (Martínez-Zapater and Salinas, 1998). For the detached leaf inoculation experiments, the plants were grown in soil at $20-25^{\circ} \mathrm{C}$ with a photoperiod of $12 \mathrm{~h}$ day/night and detached leaves of 28-30 days-old seedlings were used for inoculation. For whole seedling inoculation experiments, the plants were sown on half-strength MS plates and were grown in a chamber at $22^{\circ} \mathrm{C}$ with a photoperiod of $12 \mathrm{~h}$ day/night and 2 weeks-old plants were used for inoculation.

\section{Microscopic Examination}

To visualize the $P$. parasitica infection structures and plant cell death, leaf, and root tissues were harvested at different time points and stained using a modified trypan blue method as described by Wang et al. (2011). The samples were mounted in $50 \%$ glycerol and viewed under Olympus BX-51 microscope equipped with differential interference contrast (DIC) optics (Olympus, Japan). For microscopic characterization of infection with $P$. parasitica transformant 1121 , which stably expresses green fluorescent protein (GFP), infected tissues were collected and viewed under Olympus BX-51 fluorescent microscope with the GFP filter (BP450-BP480).

\section{Callose Deposition Staining}

To visualize callose deposition, the $A$. thaliana leaf tissues were stained with aniline blue (Bouwmeester et al., 2011). After inoculation, the tissues were cleared overnight in $96 \%$ ethanol and stained with $1 \%(\mathrm{w} / \mathrm{v})$ aniline blue in $150 \mathrm{mM}$ $\mathrm{K}_{2} \mathrm{HPO}_{4}(\mathrm{pH}$ 9.5) for $1 \mathrm{~h}$. The samples were mounted on glass microscope slides in 50\% glycerol and viewed under Olympus BX-51 fluorescent microscope with the UV filter (BP330-BP385).

\section{Association Mapping of RPPA1 ${ }^{Z u-1}$ Using SLAF-seq}

Two phenotypically contrasting bulks each comprising $30 \mathrm{~F}_{2}$ plants, one resistant and the other susceptible to $P$. parasitica were generated. Including the parent pools, we totally sequenced four pools for determination of the regions associated with resistance phenotype. The raw sequencing data have been deposited to the Sequenced Read Archive (Bioproject number: PRJNA282643). The accession numbers for the parent accessions $\mathrm{Zu}-1$ and Ler-1, and the resistant and susceptible bulks are SRR2002811, SRR2002808, SRR2002809, and SRR2002285, respectively. The specific-locus amplified fragment sequencing (SLAF-seq) procedure (Biomarker, Beijing, China) was performed as described (Sun et al., 2013). For genome-wide association analysis, all the markers were parsed as multiple contingency tables and Fisher's exact tests were used to calculate the associations of the makers with the resistance locus. Multiple tests were controlled by false discovery rate using a Bonferroni procedure as developed in the R program (http://www.r-project. org). 


\section{Results}

\section{Phenotypic Variations in Arabidopsis thaliana to Phytophthora parasitica Pp016}

In a previous study (Wang et al., 2011), we examined the interaction phenotypes of $A$. thaliana accessions to $P$. parasitica strain Pp016. By using root inoculation method, almost all of the $25 \mathrm{~A}$. thaliana accessions were susceptible. However, by infection of detached leaves, some of the 20 briefly examined accessions exhibited varying degrees of resistance. To further investigate the resistance on the leaves of $A$. thaliana response to $P$. parasitica, we examined additional 28 accessions by inoculation of detached leaves with $P$. parasitica Pp016, and examined the extent of disease development macroscopically and microscopically 3 days post inoculation (dpi). The results showed that the 48 (including 20 accessions briefly examined in previous study) accessions (Table 1) tested can be categorized into four distinct groups $(\mathrm{N}, \mathrm{Y}, \mathrm{W}, \mathrm{H})$ according to the resistance response, disease severity scored with water-soaked lesion size, development of abundant haustoria, and production of sporangia. The interaction phenotypes were characterized as follows:

Phenotype N (Figure 1A): healthy leaves with no visible disease symptoms and nearly no pathogen colonized at the inoculation site (Figure 1B), but Necrotic flecks on the leaf surface observed under a dissecting microscope at low magnification (Figure 1I). Phenotype Y (Figure 1C): Yellowing surrounds inoculation sites within one-third of leaf sizes with spare hyphae (Figure 1D) and occasional visible haustoria observed. Phenotype W (Figure 1E): Water-soaked lesions on leaves with heavy hyphae colonized (Figure 1F) and numerous haustoria formed $3 \mathrm{dpi}$, and by $4 \mathrm{dpi}$ abundant sporangia formed on the leaf surface as described (Wang et al., 2011). Phenotype H (Figure 1G): Hyper-susceptible phenotype exhibited the whole leaves watered with heavier hyphae colonized (Figure 1H) and extremely abundant haustoria (Figure 1J) formed $3 \mathrm{dpi}$, and also the sporangia formed earlier than phenotype $\mathrm{W}$ on the leaf surface.

Accession $\mathrm{Zu}-1$ exhibited the most resistance to Pp016, and was classified as the phenotype $\mathrm{N}$. The leaves remained green,

TABLE 1 | Phenotypic variation of Arabidopsis thaliana accessions (48) to Phytophthora parasitica strain Pp016.

\begin{tabular}{|c|c|}
\hline Phenotypes & Accessions \\
\hline N & $\mathrm{Zu}-1^{\#}$ \\
\hline Y & $\begin{array}{l}\text { Bla-11, Et-0, Gre-0, Lz-0, Ost-0, Tsu-1 } \\
(\text { Di-1, Is-0, Mc-0) }\end{array}$ \\
\hline w & $\begin{array}{l}\text { Aa-0, Ang-1, Bl-1, Chi-0, Cit-0, Do-0, Fi-0, Ge-0, Gie-0, In-0, } \\
\text { Je-0, Mr-0, No-0, Nok-0, Per-2, Rou-0, Rsch-0, Ru-0, Su-0, } \\
\text { Tul-0, Uk-1, Zu-0 } \\
\text { (Bs-1, Co-1, Col-0, Er-0, Fe-1, Lo-1, Ms-0, Mt-0, Old-1, Pla-2, } \\
\text { Sap-0, Sg-1, Sorbo)" }\end{array}$ \\
\hline $\mathrm{H}$ & Ler\#, Nd-O ${ }^{\#}, \mathrm{Sf}-1^{\#}$ \\
\hline
\end{tabular}

Leaves of 4 weeks-old A. thaliana seedlings were assessed for susceptibility by inoculation with P. parasitica Pp016 zoospore suspension ( $1 \times 10^{5}$ zoospores $\left./ \mathrm{mL}\right)$, and the results were scored 3 dpi; " accessions tested in previous study Wang et al., 2011). similar to the control leaves for up to $6 \mathrm{dpi}$. Nine of 48 accessions were moderately resistant, being classified as the phenotype $\mathrm{Y}$, with restricted water-soaked lesions but smaller than one-third of leaf sizes by 3 dpi. Most A. thaliana accessions (35 of 48) were susceptible to Pp016 (scored in this study as the phenotype W), similar to the accession Col-0 as described (Wang et al., 2011). Three accessions, including Ler, were the most susceptible to Pp016 (phenotype H), with whole leaf water-soaked and heavily colonized by 3 dpi.

\section{Zu-1 is Highly Resistant to a Set of Diverse $P$. parasitica Strains}

Of the 48 accessions tested, $\mathrm{Zu}-1$ exhibited the most resistant phenotype to $\mathrm{Pp} 016$. To investigate whether the $\mathrm{Zu}-1$ resistance phenotype is broad-spectrum, we performed infection assays with additional $19 P$. parasitica strains isolated from different host plants (Table 2). The results indicated that $\mathrm{Zu}-1$ is highly resistant to all of examined strains. Accession Ler is susceptible to all the strains except Pp008 and Pp009, and hyper-susceptible to a half of strains tested. Accession Col-0 exhibited different phenotypes to the 20 examined $P$. parasitica strains.

\section{Microscopic Characterization of Resistance in Zu-1 to $P$. parasitica}

To further understand the resistance of $\mathrm{Zu}-1$ to $P$. parasitica, we inoculated $\mathrm{Zu}-1$ and the susceptible accession Ler with zoospores of $P$. parasitica strain Pp016 and examined for cellular reactions at different time points microscopically, after the infected tissues were stained with trypan blue. As shown in Figure 2, at the initial 3 hours post inoculation (hpi), there were no differences between $\mathrm{Zu}-1$ and Ler in response to $P$. parasitica infection, including the timing of cyst germination, the rates of cyst germination and development of appressoria (Figures 2A-C). However, starting from when the penetration pegs emerged beneath the appressoria and the penetration hyphae started to grow between the anticlinal walls of two epidermal cells, differences between $\mathrm{Zu}-1$ and Ler became apparent. In the susceptible Ler plants, the penetration process was similar to Col-0 as described (Wang et al., 2011). However, many more haustoria formed and abundant sporangia produced earlier in Ler than that in Col-0, which is indicative of heavy colonization by strain $\mathrm{Pp}$ 016. In resistant $\mathrm{Zu}-1$ plants, the earliest microscopically visible response was observed at $6 \mathrm{hpi}$, when cysts germinated and developed appressoria. At the attempted penetration sites of Zu-1 by Pp016, papilla appeared with heavy deposition of callose materials (Figures 2D-F), as revealed by aniline blue staining. Mostly, the wall of some epidermal cells was encased with callose (Figures 2G,H). HR is the most important phenotype in the resistant plants against pathogens. Typically, only a few epidermal cells that were penetrated by Pp016 showed a cell death response. This was frequently followed by, at nearly all the infection sites, rapid HRs, which limited in single or a few epidermal cells (Figure 2I), or stoma guard cells (Figure 2J), or stoma guard cells and an adjacent epidermal cells (Figure $2 \mathbf{K}$ ). This necrotic response typically stops $P$. parasitica infection (Figure 2L). 

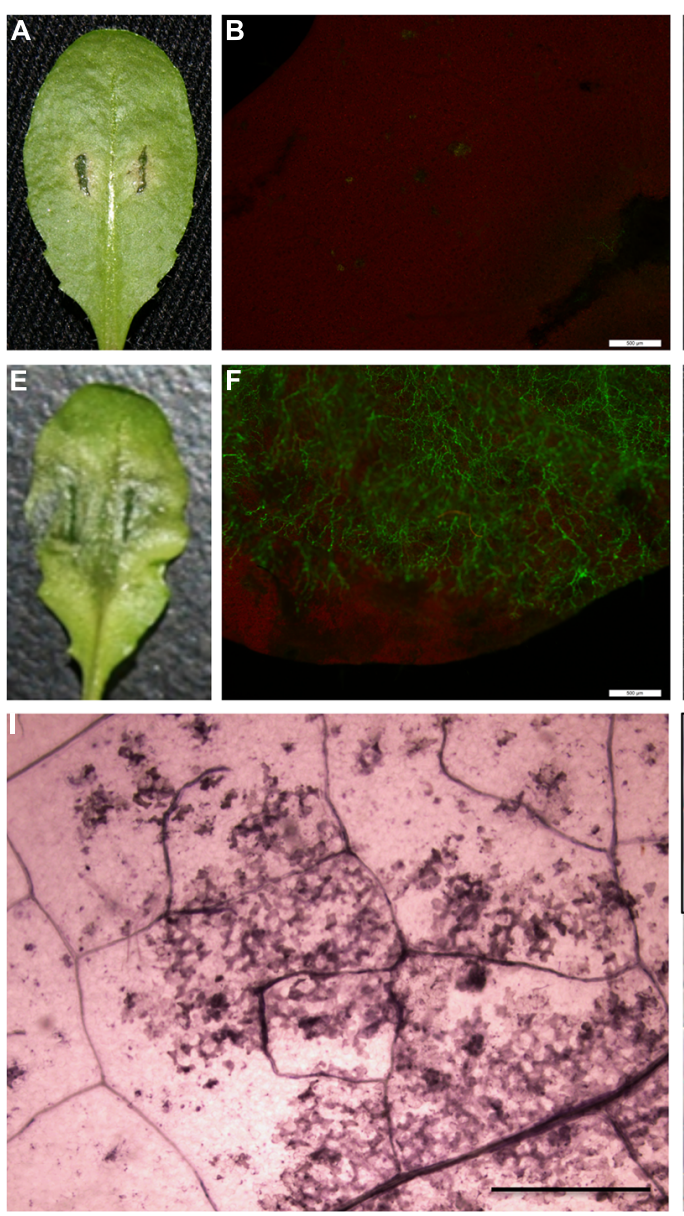

FIGURE 1 | Variation of interaction phenotypes of Arabidopsis thaliana accessions inoculated with Phytophthora parasitica strain Pp016. Leaves of 4 weeks-old $A$. thaliana seedlings were inoculated with zoospores of $P$. parasitica strain Pp016 $\left(1 \times 10^{5}\right.$ zoospores $\left./ \mathrm{mL}\right)$ and the phenotype scored 3 days post inoculation (dpi). To access the extent of pathogen infection, P. parasitica transformant 1121 that stably expresses green fluorescent protein (GFP) was used for inoculation. (A) Phenotype N, no visible symptom on the leaf surface $(Z u-1)$. (C) Phenotype $Y$, yellowish
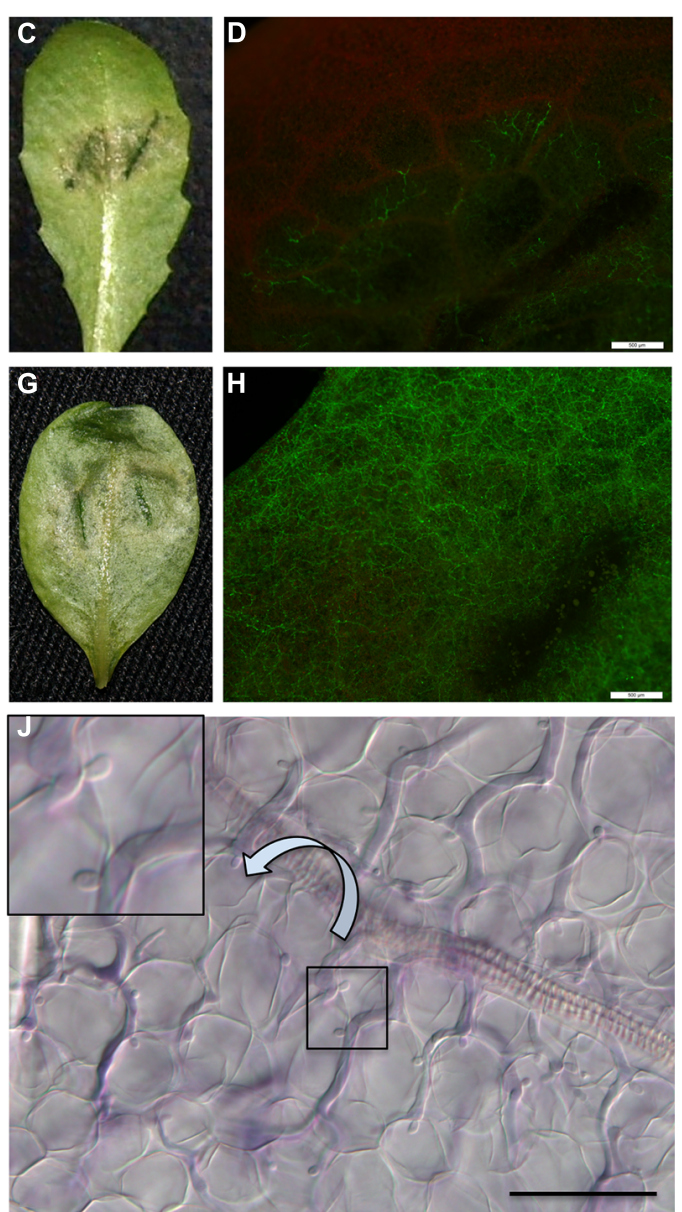

around the restricted water-soaked lesions that are smaller than one-third leaf sizes (Et-0). (E) Phenotype W, water-soaked lesions (Col-0).

(G) Phenotype $\mathrm{H}$, hyper-susceptible with the whole leaves water-soaked (Ler). (B,D,F,H) Cytological characterization of hyphae colonization in (A,C,E,G), respectively, by P. parasitica strain 1121 (bar, $500 \mu \mathrm{m})$.

(I) Necrotic flecks were observed under a dissecting microscope at low magnification $(4 \times)$ (bar, $500 \mu \mathrm{m})$. (J) Extremely abundant haustoria formed in mesophyll cells in the phenotype $H$ (bar, $50 \mu \mathrm{m}$ ).

\section{Zu-1 is Susceptible to Root Infection by $P$. parasitica}

Since $P$. parasitica is a typical root pathogen of many plants, we also tested whether $\mathrm{Zu}-1$ is resistant to root infection. Live root tissues were inoculated by dipping into a zoospore suspension. The result showed that $\mathrm{Zu}-1$ is susceptible to $P$. parasitica, with the inoculated seedlings wilted and collapsed $4 \mathrm{dpi}$. Compared with Ler and Col-0, $\mathrm{Zu}-1$ roots exhibited no clear differences to $P$. parasitica infection. The microscopic characterization showed that the infection process by $P$. parasitica on $\mathrm{Zu}-1$ was similar to that on Ler and Col-0. Zoospores geminated $1.5 \mathrm{hpi}$ and nearly all zoospores developed germ tubes (Figure $\mathbf{2 M}$ ) at $6 \mathrm{hpi}$. Colonization by invasive hyphae was observed both inside the cells and in the intercellular spaces of the root tissues, and haustoria-like structures (Figure 2N) were formed in the cortex at $12 \mathrm{hpi}$. And in many cases, the haustoria-like structures in roots were as wide as the hyphae they originated from (Figure 20). By 48 hpi, heavy $P$. parasitica hyphae colonization in root tissues was apparent (Figure 2P) and, by $72 \mathrm{hpi}$, numerous sporangia were visible (Figure 2Q).

\section{Genetic Analysis of Resistance in $\mathrm{Zu}-1$ to $P$ parasitica}

To examine inheritance of resistance in $\mathrm{Zu}-1$, genetic crosses were carried out using $\mathrm{Zu}-1$ and the fully susceptible Ler. The obtained $25 \mathrm{~F}_{1}$ plants and the $\mathrm{F}_{2}$ populations (Table 3) were inoculated with $\mathrm{Pp} 016$ and scored for response phenotypes 3 dpi. The results showed that $25 \mathrm{~F}_{1}$ progenies exhibited a moderate resistant response (Phenotype $\mathrm{Y}$ ) with some phenotypic variations to $\mathrm{Pp} 016$. Compared with the parents $\mathrm{Zu}-1$ (Figure 1A) and Ler (Figure 1C), the water-soaked lesions of $F_{1}$ progenies were restricted, generally within 
TABLE 2 | Phenotypic characterization of $A$. thaliana accessions Zu-1, Ler, and Col-0 to $20 P$. parasitica strains.

\begin{tabular}{|c|c|c|c|c|c|}
\hline \multicolumn{3}{|c|}{ Phytophthora parasitica strains } & \multicolumn{3}{|c|}{ Arabidopsis accessions } \\
\hline Strains & Host plant & Mating type & $\mathrm{Zu}-1$ & Ler & Col-0 \\
\hline Pp004 & Tobacco & A1 & $\mathrm{N}$ & $\mathrm{H}$ & $\mathrm{Nd}$ \\
\hline Pp008 & Tobacco & A2 & $\mathrm{N}$ & $\mathrm{N}$ & $\mathrm{N}$ \\
\hline Pp009\# & Tobacco & $\mathrm{A} 2$ & $\mathrm{~N}$ & $\mathrm{~N}$ & $\mathrm{~N}$ \\
\hline Pp010 & Pawpaw & $\mathrm{A} 2$ & $\mathrm{~N}$ & $\mathrm{H}$ & W \\
\hline Pp012 & Pawpaw & $\mathrm{A} 2$ & $\mathrm{~N}$ & W & Y \\
\hline Pp014 & Tobacco & $\mathrm{A} 2$ & $\mathrm{~N}$ & W & $\mathrm{H}$ \\
\hline Pp016 $\#$ & Tobacco & $\mathrm{A} 1$ & $N$ & $\mathrm{H}$ & W \\
\hline $\mathrm{Pp} 017$ & - & $\mathrm{A} 2$ & $\mathrm{~N}$ & W & W \\
\hline Pp018 & Banksia species & $\mathrm{A} 2$ & $N$ & $\mathrm{H}$ & W \\
\hline Pp019 & - & $\mathrm{A} 2$ & $N$ & W & $\mathrm{Nd}$ \\
\hline Pp020 & Banksia species & $\mathrm{A} 2$ & $\mathrm{~N}$ & $\mathrm{H}$ & $\mathrm{Nd}$ \\
\hline Pp022 & Citrus species & A1 & $N$ & $\mathrm{H}$ & Y \\
\hline Pp023 & Citrus species & A1 & $N$ & W & Y \\
\hline Pp024 & Citrus species & $\mathrm{A} 1$ & $\mathrm{~N}$ & $\mathrm{H}$ & Y \\
\hline Pp025\# & Dendrobium candidum & $\mathrm{A} 2$ & $\mathrm{~N}$ & $\mathrm{H}$ & $\mathrm{H}$ \\
\hline Pp026 & D. candidum & $\mathrm{A} 2$ & $\mathrm{~N}$ & $\mathrm{H}$ & $\mathrm{Nd}$ \\
\hline Pp028 & D. candidum & $\mathrm{A} 2$ & $N$ & W & $\mathrm{Nd}$ \\
\hline Pp029 & D. candidum & $\mathrm{A} 2$ & $N$ & W & $\mathrm{Nd}$ \\
\hline Рp030 & D. candidum & A2 & $\mathrm{N}$ & W & $\mathrm{Nd}$ \\
\hline Pp031 & Tobacco & - & $\mathrm{N}$ & $\mathrm{H}$ & W \\
\hline
\end{tabular}

Leaves of 4 weeks-old A. thaliana seedlings were assessed for susceptibility by inoculation with P. parasitica zoospore suspension $\left(1 \times 10^{5}\right.$ zoospores $\left./ \mathrm{mL}\right)$, and the results were scored 3 days post inoculation; nd, not determined; \# strains used in a previous study (Wang et al., 2011).

(Figure 3A) or a little more than one-third of leaf sizes (Figure 3B). The surrounding leaf tissues became yellowish $3 \mathrm{dpi}$, but the colonizing hyphae were restricted within the inoculation sites. The extent of $P$. parasitica colonization in $\mathrm{F}_{1}$ progenies with moderately resistant response was also examined microscopically. The invasive hyphae observed were sparse (Figure 3C). Compared with abundant haustoria formed in Ler (Figure 1J), fewer haustoria were formed in the mesophyll cells from the intercellular penetrating hyphae in $F_{1}$ progenies (Figure 3D). Numerous cell death in the epidermal cells also occurred (Figure 3E) but not as many as observed for the parent $\mathrm{Zu}-1$, in which nearly all the penetrated epidermal cells responded with cell death.

Segregation analysis of $\mathrm{F}_{2}$ populations showed that one quarter (47/176) were susceptible to Pp016, similar to Ler, while another quarter $(42 / 176)$ were similar to $\mathrm{Zu}-1$ being resistant to Pp016 (Table 3). Nearly half (87/176) of $F_{2}$ populations showed the $\mathrm{F}_{1}$ phenotype, being moderately resistant. The segregation $(\mathrm{N}$ : $\left.\mathrm{Y}: \mathrm{S}^{*}\right)$ ratio was very close to $1: 2: 1\left(\chi^{2}=0.307, P=0.8578\right)$ in $\mathrm{F}_{2}$ populations, consistent with the interpretation that a single semi-dominant locus confers resistance in $\mathrm{Zu}-1$ to $P$. parasitica Pp016. The locus is designated as $R P P A 1^{\mathrm{Zu}-1}$ (Resistance to $P$. parasitica 1). Also, inoculation of the $F_{2}$ populations with another $P$. parasitica strain Pp025 revealed 100\% correlation of phenotypes that was observed with strain Pp016. This indicates that the RPPA $1^{Z u-1}$ locus confers resistance to at least two strains of $P$. parasitica.

\section{Preliminary Mapping of RPPA1 ${ }^{Z u-1}$ Using SLAF-seq}

By employing SLAF-seq method (Sun et al., 2013), we sequenced four samples, including two parents and two $F_{2}$ bulked populations. As the $\mathrm{F}_{2}$ populations were obtained by selfing the $\mathrm{F}_{1}$ progeny of a cross between two fully homozygous parents with the genotypes RR or rr, a total of 2742 polymorphic SLAFs were detected and were analyzed for the association with resistance in accession $\mathrm{Zu}-1$ to $P$. parasitica strain Pp016. The results further showed that the strongest associated region was located between 7.1 and 11.2 Mb in the chromosome IV (Figure 4).

\section{Discussion}

Elucidation of the mechanism of the interaction between the pathogen and host plants has been the focus for understanding of disease resistance. The objective of this research is to investigate phenotypic and genetic variations in $A$. thaliana to $P$. parasitica. In this paper, we inoculated 28 A. thaliana accessions with $P$. parasitica strain Pp016 and variation in resistance phenotypes was evident. Based on careful assessment of variation in resistance phenotypes to $P$. parasitica strain Pp016, the 48 accessions can be divided into four distinct groups. We found that most accessions (38/48) are susceptible to Pp016, including three hyper-susceptible accessions. Nine of 48 accessions are resistant. We also showed that $\mathrm{Zu}-1$ is highly resistant to all 20 strains tested. The genetic analysis of segregating populations derived from cross $\mathrm{Zu}-1$ and Ler showed that the resistance to at least two of $P$. parasitica strains is conditioned by a single semi-dominant locus.

A. thaliana accession $\mathrm{Zu}-1$ is resistant to $20 \mathrm{P}$. parasitica strains isolated from different host plants, including tobacco, pawpaw, Banksia species, Citrus species, and Dendrobium candidum. Apart from the origin the $P$. parasitica strains were isolated, the strain selection was also based on their great differences in virulence spectrum on a set of 12 tobacco cultivars (data not shown). For example, strain Pp014 is virulent to all 12 tobacco cultivars, Pp009 is virulent to only three of them, and Pp016 is virulent to eight of them.

Zu-1 was highly resistant to all examined $P$. parasitica strains and microscopically exhibited strong cell death response. Resistance in $\mathrm{Zu}-1$ was manifested as necrosis of plant cells in the epidermal layer (Figures 2I-K), which comprised only one or a few epidermal cells, and the pathogen was not observed in the mesophyll cells, which is different from the resistance phenotype of Col-0 in response to infection with P. parasitica strain Pp009 (Wang et al., 2011), and other Phytophthora pathogens on A. thaliana (Roetschi et al., 2001; Wang et al., 2013), in which the mesophyll cells undergoing HR to stop the penetration of progressive hyphae. The diverse range of reaction phenotypes of different plant-pathogen combinations also occurs in A. thaliana to the oomycete pathogen Hyaloperonospora arabidopsidis (Koch and Slusarenko, 1990; Parker et al., 1993; Reignault et al., 1996). The different responses of known resistance were due to the presence of different resistance loci. The thickening of the cell wall and the formation of callose-containing papillae 


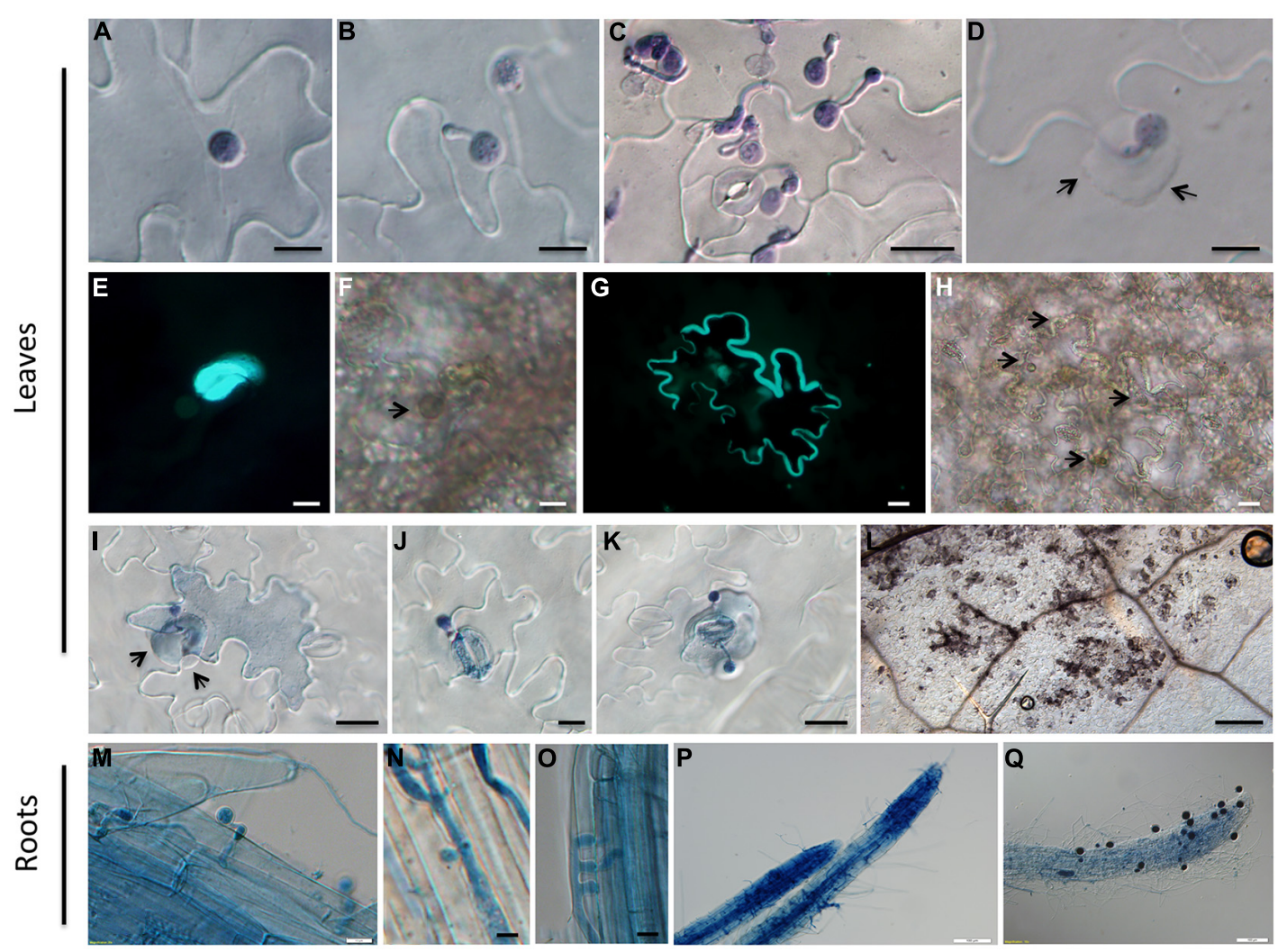

FIGURE 2 | Cytological characterization of infection of $\boldsymbol{A}$. thaliana accession Zu-1 to $P$. parasitica Pp016. (A) Zoospores encysted at $30 \mathrm{~min}$ (bar, $10 \mu \mathrm{m}$ ). (B) Cyst germinated with a germ tube at $1.5 \mathrm{hpi}$ (bar, $10 \mu \mathrm{m}$ ). (C) Cyst germinated and appressorium formed at $3 \mathrm{hpi}$ (bar, $20 \mu \mathrm{m})$. (D) Heavy deposition of materials surrounding the attempted penetration sites (arrows) at the border of two anticlinal walls of epidermal cells (bar, $10 \mu \mathrm{m}$ ).

(E) Fluorescence of callose at the penetration sites along the junction between epidermal cell walls (bar, $10 \mu \mathrm{m}$ ). (F) Bright-field image of (E), showing a germinated cyst at the penetration site (bar, $10 \mu \mathrm{m})$. Arrows point to the place where the cyst is visible. (G) Fluorescence and thickening of the cell wall showing an infected epidermal cell (bar, $20 \mu \mathrm{m})$. (H) Bright-field image of
(G) showing the germinated cysts at the penetration sites (bar, $20 \mu \mathrm{m}$ ). Arrows point to places where the cysts are visible. (I-K) Hypersensitive response (HR) of an epidermal cell (bar, $20 \mu \mathrm{m}$ ), stoma guard cells (bar, $10 \mu \mathrm{m})$, and an epidermal cell close to the stoma (bar, $20 \mu \mathrm{m}$ ), in response to penetration by Pp016 at 48 hpi. Arrows indicate the heavy deposit of material surrounding the attempted penetration site in epidermal cells. (L) Necrosis on the leaf surface $72 \mathrm{hpi}$ and the hypersensitive cells are stained as darker color (bar, $200 \mu \mathrm{m})$. (M) Zoospores germinated and formed appressoria at $6 \mathrm{hpi}$ (bar, $10 \mu \mathrm{m})$. (N,O) Haustoria-like structures developed in the cortex at $12 \mathrm{hpi}(\mathrm{bar}, 10 \mu \mathrm{m})$. (P) Heavy hyphal colonization in roots at 48 hpi (bar, $100 \mu \mathrm{m})$. (Q) Sporangia produced on the root surface at $72 \mathrm{hpi}$ (bar, $100 \mu \mathrm{m})$.
TABLE 3 | Genetic analysis of resistance in $A$. thaliana $\mathrm{Zu}-1$ to $P$. parasitica Pp016.

\begin{tabular}{llll}
\hline Plant type & Phenotype & Plants tested & Symptom \\
\hline Zu-1 & $\mathrm{N}$ & 20 & $20, \mathrm{~N}$ \\
Ler & $\mathrm{H}$ & 20 & $20, \mathrm{H}$ \\
$\mathrm{F}_{1}$ & $\mathrm{Y}(\mathrm{Zu}-1$ as female) & 17 & $17, \mathrm{Y}$ \\
& $\mathrm{Y}($ Ler as female $)$ & 8 & $8, \mathrm{Y}$ \\
$\mathrm{F}_{2}$ & Segregating & 176 & $42, \mathrm{~N}$ \\
& & & $87, \mathrm{Y}$ \\
& & & $47, \mathrm{H}$ \\
\hline
\end{tabular}

were evident in $\mathrm{Zu}-1$, which were common characteristics in other plant resistance responses (Figures 2D-H). Massive callose depositions were observed at the penetration sites and in the cells undergoing HR (Figure 2I). These results suggest that resistance in $\mathrm{Zu}-1$ is triggered at early stage of infection. In addition, of the described A. thaliana and Phytophthora interactions (Wang et al., 2011, 2013), the failure of the first pathogen penetration often leads to the development of secondary germ tubes and appressoria. Interestingly, we did not observe this phenomenon in $\mathrm{Zu}-1$. Furthermore, at the initial $6 \mathrm{hpi}$, cyst germination and appressorial development were similar to that on the susceptible Ler.

$\mathrm{Zu}-1$ is susceptible to root infection by $P$. parasitica strain Pp016, which is very different from its highly resistant to leaf infection. Actually, with root inoculation method, most accessions of $A$. thaliana cannot prevent the $P$. parasitica pathogen (Attard et al., 2010; Wang et al., 2011). It is evident that different plant organs may elicit the activation of specific signaling networks. Organ-specificity of defense responses in plant disease has been described (Hermanns et al., 2003; Rookes et al., 2008). A particular pathogen only infects some organs of a genetically susceptible host but not other organs or the entire plant. As described in many plant-pathogen interactions, the 

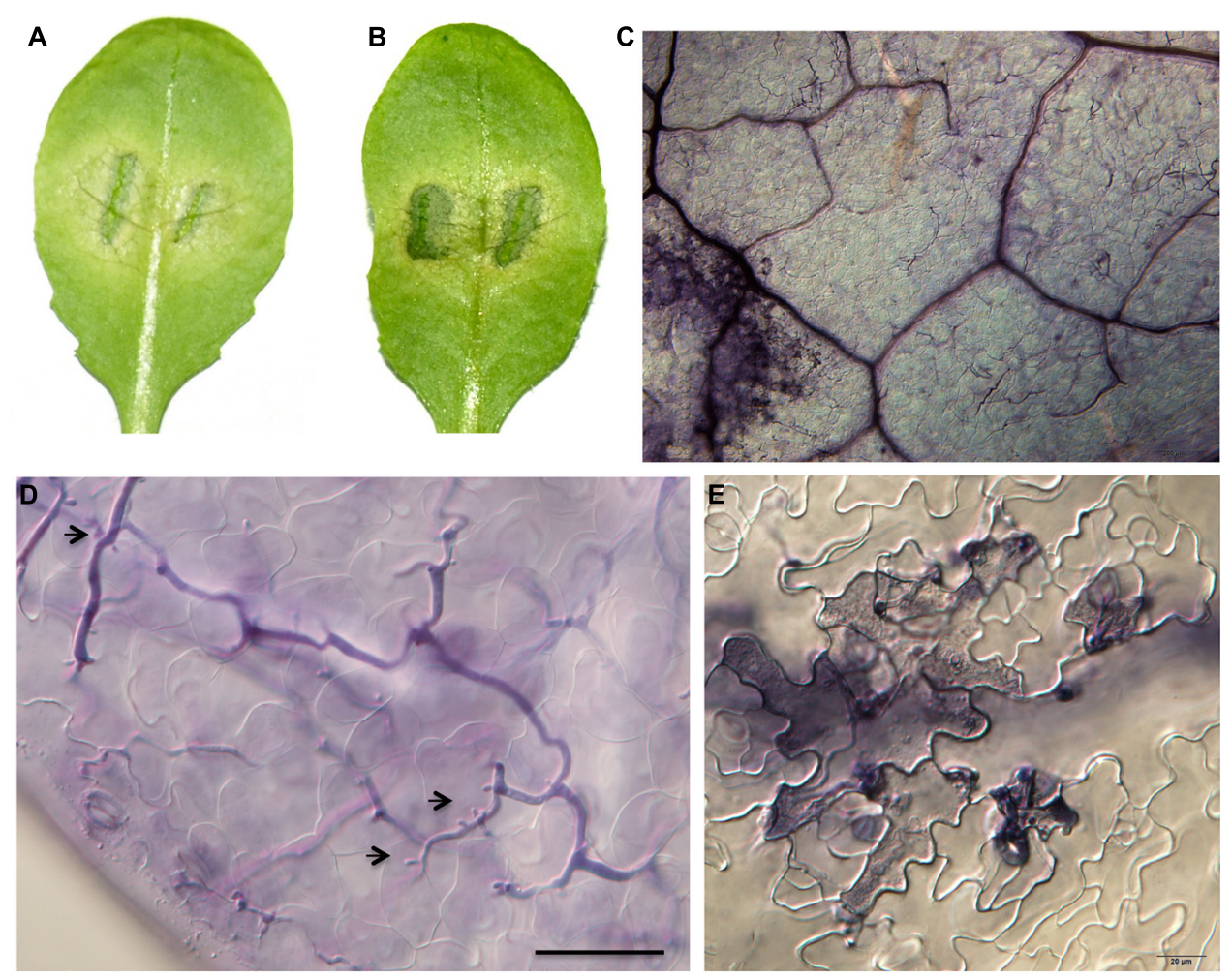

FIGURE 3 | Phenotypic characterizations of $F_{1}$ progenies of $\mathrm{Zu}-1$ and Ler to infection by $P$. parasitica strain Pp016. Leaves of 4 weeks-old A. thaliana seedlings were inoculated with zoospores of $P$. parasitica strain Pp016 ( $1 \times 10^{5}$ zoospores $\left./ \mathrm{mL}\right)$ and the phenotype scored 3 dpi. $(\mathbf{A}, \mathbf{B})$ Leaves of $F_{1}$ progenies are moderately resistant with restricted water-soaked lesions. (C) Less colonized hyphae in epidermal cells (bar, $200 \mu \mathrm{m}$ ). (D) Less colonized hyphae in mesophyll cells with few haustoria (bar, $50 \mu \mathrm{m}$ ). Arrows indicate haustoria-like structures. (E) HR in epidermal cells (bar, $20 \mu \mathrm{m})$.

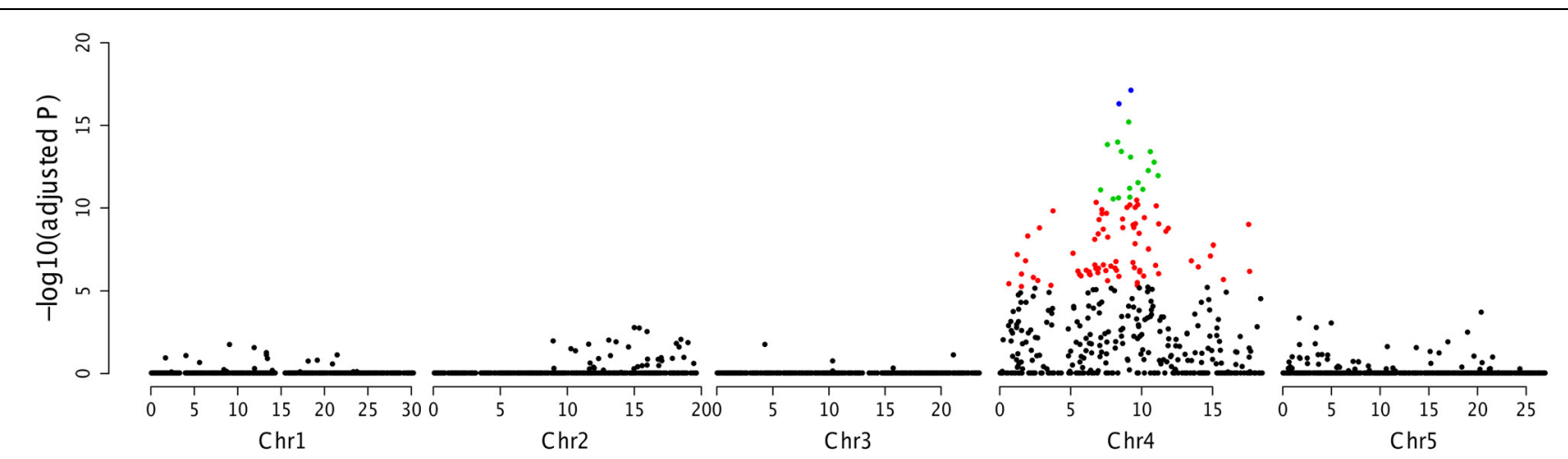

FIGURE 4 | Association mapping analysis of RPPA1 ${ }^{\text {Zu-1 }}$ by SLAF-seq. The adjusted $P$ values for the markers associated with the resistance locus were showed in a logarithmic scale.

plant defense hormones salicylic acid (SA), jasmonic acid (JA), and ethylene (ET) have been shown to play important roles in the resistance reaction. SA-signaling is important for defense against biotrophic pathogens, while JA and/ or ET-signaling is involved in defense against necrotrophic pathogens, although there are exceptions and additional complexities (Glazebrook, 2005). During root infection by $P$. parasitica, SA, JA, and ET signaling pathways cooperate in the defenses (Attard et al.,
2010), which is similar with Arabidopsis defense response against the root fungal pathogen Fusarium oxysporum (Berrocal-Lobo and Molina, 2008), but is different from other Phytophthora pathogens on Arabidopsis (Roetschi et al., 2001; Rookes et al., 2008; Schlaeppi et al., 2010; Wang et al., 2013). However, most of the results were obtained from the leaf infections. The underlying mechanisms mediating differences of defense pathways in host plants and the organ-specificity are complicated. $\mathrm{Zu}-1$ exhibited 
obvious differences in resistance to $P$. parasitica and may provide an excellent model for understanding organ-specific disease resistance.

Since semi-dominance is a widespread characteristic of resistance genes and the mechanistic implications have been discussed (Crute and Norwood, 1986). For example, the first resistance gene RPP5 from $A$. thaliana to $H$. arabidopsidis was identified as a semi-dominant gene, which resulted in resistance in the heterozygote being lower than in the resistant homozygote (Parker et al., 1993, 1997). In our observation, the moderate resistant phenotype of $F_{1}$ plants of $\mathrm{Zu}-1$ and Ler exhibits yellowish characters macroscopically and restricted lesion development. In addition, the $F_{2}$ populations were observed with a ratio very close to $1: 2: 1 \quad\left(\chi^{2}=0.307\right.$, $P=0.8578$ ). Based on the phenotype of $\mathrm{F}_{1}$ progenies and the $\mathrm{F}_{2}$ segregation data, the resistance in $\mathrm{Zu}-1$ to $P$. parasitica $\mathrm{Pp} 016$ seemed to be conditioned by a semi-dominant locus, $R P P A 1^{Z u-1}$. However, the heterozygous $R P P A 1^{Z u-1}$ in $\mathrm{F}_{2}$ plants to $P$. parasitica exhibited intermediate phenotype, making it difficult to achieve fine physical mapping of the RPPA $1^{Z u-1}$ locus. Analysis of selected $\mathrm{F}_{3}$ progenies would be necessary to assign accurately a genotype of the plants.

Recent developments in high throughput next-generation sequencing technologies now can provide new strategies for sequence-based genotyping. Several methods have been developed that involve sequencing only a small fraction of the entire genome. Specific-locus amplified fragment sequencing (SLAF-seq) approach is a strategy developed for the de novo SNP discovery and genotyping of large populations using an enhanced RRL sequencing method (Sun et al., 2013). And this method has been used for haplotype mapping, genetic mapping, linkage mapping, and polymorphism mapping (Chen et al., 2013; Sun et al., 2013; Zhang et al., 2013; Chen et al., 2014). In this study, we employed the SLAF-seq approach to map the locus

\section{References}

Ali, S., and Bakkeren, G. (2011). Fungal and oomycete effectors-strategies to subdue a host. Can. J. Plant Pathol. 33, 425-446. doi: 10.1080/07060661.2011.625448

Allen, R. L., Bittner-Eddy, P. D., Grenville-Briggs, L. J., Meitz, J. C., Rehmany, A. P., Rose, L. E., et al. (2004). Host-parasite coevolutionary conflict between Arabidopsis and downy mildew. Science 306, 1957-1960. doi: 10.1126/science.1104022

Antonopoulos, D. F., Melton, T., and Mila, A. L. (2010). Effects of chemical control, cultivar resistance, and structure of cultivar root system on black shank incidence of tobacco. Plant Dis. 94, 613-620. doi: 10.1094/PDIS-945-0613

Arabidopsis Genome Initiative [AGI]. (2000). Analysis of the genome sequence of the flowering plant Arabidopsis thaliana. Nature 408, 796-815. doi: $10.1038 / 35048692$

Attard, A., Gourgues, M., Callemeyn-Torre, N., and Keller, H. (2010). The immediate activation of defense responses in Arabidopsis roots is not sufficient to prevent Phytophthora parasitica infection. New Phytol. 187, 449-460. doi: 10.1111/j.1469-8137.2010.03272.x.

Bailey, K., Çevik, V., Holton, N., Byrne-Richardson, J., Sohn, K. H., Coates, M., et al. (2011). Molecular cloning of ATR $5^{\text {Emoy2 }}$ from Hyaloperonospora arabidopsidis, an avirulence determinant that triggers RPP5-mediated defense in Arabidopsis. Mol. Plant Microbe Interact. 24, 827-838. doi: 10.1094/MPMI12-10-0278
$R P P A 1^{Z u-1}$. Association analysis preliminarily determined the $R P P A 1^{Z u-1}$ locus in a region between 7.1 and $11.2 \mathrm{Mb}$ in the chromosome IV.

Phenotypic variation was characterized among the interaction with respect to the extent of pathogen colonization and the host response. The timing and degree of pathogen infection and colonization vary among the combinations. HRs range from occasionally in some small regions to almost each infection epidermal cells. The observed phenotypic variations provide a useful resource for investigating molecular process in the interaction between $A$. thaliana and $P$. parasitica. The primary identification and future cloning of the $R P P A 1^{Z u-1}$ locus may offer a valuable model for understanding the broad-spectrum resistance in plants against oomycete pathogens.

\section{Author Contributions}

Conceived and designed the experiments: WS; Performed the experiments: YM YH, QZ, and GH; Analyzed the data: WS, YM, QW, and JJ; Contributed reagents/materials/analysis tools: YM, YH, QZ, GH, JQ, and QW; Wrote the paper: YM and WS, with contribution from all authors.

\section{Acknowledgments}

We thank Dr. Cai-Zhong Jiang of University of California at Davis for providing the A. thaliana stocks, Professor Adrienne Hardham of Australian National University and Dr. Jingze Zhang of Zhejiang University for $P$. parasitica strains. We also thank Dr. Wei Liu for useful suggestions and discussions. This work was supported by National Natural Science Foundation of China (\#31125022) and China Agriculture Research System (CARS-10).

Beakes, G. W., Glockling, S. L., and Sekimoto, S. (2012). The evolutionary phylogeny of the oomycete "fungi." Protoplasma 249, 3-19. doi: 10.1007/s00709-011-0269-2

Berrocal-Lobo, M., and Molina, A. (2008). Arabidopsis defense response against Fusarium oxysporum. Trends Plant Sci. 13, 145-150. doi: 10.1016/j.tplants.2007.12.004

Blair, J. E., Coffey, M. D., Park, S.-Y., Geiser, D. M., and Kang, S. (2008). A multi-locus phylogeny for Phytophthora utilizing markers derived from complete genome sequences. Fungal Genet. Biol. 45, 266-277. doi: 10.1016/j.fgb.2007.10.010

Borhan, M. H., Gunn, N., Cooper, A., Gulden, S., Tör, M., Rimmer, S. R., et al. (2008). WRR4 encodes a TIR-NB-LRR protein that confers broad-spectrum white rust resistance in Arabidopsis thaliana to four physiological races of Albugo candida. Mol. Plant Microbe Interact. 21, 757-768. doi: 10.1094/MPMI21-6-0757

Borhan, M. H., Holub, E. B., Kindrachuk, C., Omidi, M., Bozorgmanesh-Frad, G., and Rimmer, S. R. (2010). WRR4, a broad-spectrum TIR-NB-LRR gene from Arabidopsis thaliana that confers white rust resistance in transgenic oilseed brassica crops. Mol. Plant Pathol. 11, 283-291. doi: 10.1111/j.13643703.2009.00599.x

Bouwmeester, K., de Sain, M., Weide, R., Gouget, A., Klamer, S., Canut, H., et al. (2011). The lectin receptor kinase LecRK-I.9 is a novel Phytophthora resistance component and a potential host target for a RXLR effector. PLoS Pathog. 7:e1001327. doi: 10.1371/journal.ppat.1001327 
Bozkurt, T. O., Schornack, S., Banfield, M. J., and Kamoun, S. (2012). Oomycetes, effectors, and all that jazz. Curr. Opin. Plant Biol. 15, 483-492. doi: 10.1016/j.pbi.2012.03.008

Büschges, R., Hollricher, K., Panstruga, R., Simons, G., Wolter, M., Frijters, A., et al. (1997). The barley Mlo gene: a novel control element of plant pathogen resistance. Cell 88, 695-705. doi: 10.1016/S0092-8674(00)81912-1

Chen, S., Huang, Z., Dai, Y., Qin, S., Gao, Y., Zhang, L., et al. (2013). The development of 7E chromosome-specific molecular markers for Thinopyrum elongatum based on SLAF-seq technology. PLoS ONE 8:e65122. doi: 10.1371/journal.pone.0065122

Chen, W., Yao, J., Chu, L., Li, Y., Guo, X., and Zhang, Y. (2014). The development of specific SNP markers for chromosome 14 in cotton using next-generation sequencing. Plant Breed. 133, 256-261. doi: 10.1111/pbr.12144

Coates, M. E., and Beynon, J. L. (2010). Hyaloperonospora arabidopsidis as a pathogen model. Annu. Rev. Phytopathol. 48, 329-345. doi: 10.1146/annurevphyto-080508-094422

Crute, I. R., and Norwood, J. M. (1986). Gene-dosage effects on the relationship between Bremia lactucae (downy mildew) and Lactuca sativa (lettuce): the relevance to a mechanistic understanding of host-parasite specificity. Physiol. Mol. Plant Pathol. 29, 133-145. doi: 10.1016/S0048-4059(86)80016-9

Daniel, R., and Guest, D. (2006). Defence responses induced by potassium phosphonate in Phytophthora palmivora-challenged Arabidopsis thaliana. Physiol. Mol. Plant Pathol. 67, 194-201. doi: 10.1016/j.pmpp.2006.01.003

Glazebrook, J. (2005). Contrasting mechanisms of defense against biotrophic and necrotrophic pathogens. Annu. Rev. Phytopathol. 43, 205-227. doi: 10.1146/annurev.phyto.43.040204.135923

Goritschnig, S., Krasileva, K. V., Dahlbeck, D., and Staskawicz, B. J. (2012). Computational prediction and molecular characterization of an oomycete effector and the cognate Arabidopsis resistance gene. PLoS Genet. 8:e1002502. doi: 10.1371/journal.pgen.1002502

Gururani, M. A., Venkatesh, J., Upadhyaya, C. P., Nookaraju, A., Pandey, S. K., and Park, S. W. (2012). Plant disease resistance genes: current status and future directions. Physiol. Mol. Plant Pathol. 78, 51-65. doi: 10.1016/j.pmpp.2012.01.002

Gutiérrez, W. A., and Mila, A. L. (2007). A rapid technique for determination of races of Phytophthora nicotianae on tobacco. Plant Dis. 91, 985-989. doi: 10.1094/PDIS-91-8-0985

Hermanns, M., Slusarenko, A. J., and Schlaich, N. L. (2003). Organ-specificity in a plant disease is determined independently of $R$ gene signaling. Mol. Plant Microbe Interact. 16, 752-759. doi: 10.1094/MPMI.2003.16.9.752

Holub, E. B., Brose, E., Tör, M., Clay, C., Crute, I. R., and Beynon, J. L. (1995). Phenotypic and genotypic variation in the interaction between Arabidopsis thaliana and Albugo candida. Mol. Plant Microbe Interact. 8, 916-928. doi: 10.1094/MPMI-8-0916

Jones, J. D. G., and Dangl, J. L. (2006). The plant immune system. Nature 444, 323-329. doi: 10.1038/nature05286

Joshi, R. K., and Nayak, S. (2013). Perspectives of genomic diversification and molecular recombination towards R-gene evolution in plants. Physiol. Mol. Biol. Plants 19, 1-9. doi: 10.1007/s12298-012-0138-2

Judelson, H. S. (2012). Dynamics and innovations within oomycete genomes: insights into biology, pathology, and evolution. Eukaryot. Cell 11, 1304-1312. doi: 10.1128/EC.00155-12

Koch, E., and Slusarenko, A. (1990). Arabidopsis is susceptible to infection by a downy mildew fungus. Plant Cell 2, 437-445. doi: 10.1105/tpc.2.5.437

Kou, Y., and Wang, S. (2010). Broad-spectrum and durability: understanding of quantitative disease resistance. Curr. Opin. Plant Biol. 13, 181-185. doi: 10.1016/j.pbi.2009.12.010

Kroon, L. P. N. M., Brouwer, H., de Cock, A. W. A. M., and Govers, F. (2012). The genus Phytophthora anno 2012. Phytopathology 102, 348-364. doi: 10.1094/PHYTO-01-11-0025

Liu, J., Liu, X., Dai, L., and Wang, G. (2007). Recent progress in elucidating the structure, function and evolution of disease resistance genes in plants. J. Genet. Genomics 34, 765-776. doi: 10.1016/S1673-8527(07)60087-3

Martínez-Zapater, J., and Salinas, J. (1998). Arabidopsis Protocols. Totowa, NJ: Humana Press. doi: 10.1385/0896033910

Meng, Y., Zhang, Q., Ding, W., and Shan, W. (2014). Phytophthora parasitica: a model oomycete plant pathogen. Mycology 5, 43-51. doi: $10.1080 / 21501203.2014 .917734$
Nishimura, M. T., and Dangl, J. L. (2010). Arabidopsis and the plant immune system. Plant J. 61, 1053-1066. doi: 10.1111/j.1365-313X.2010.04131.x

Parker, J. E., Coleman, M. J., Szabò, V., Frost, L. N., Schmidt, R., Van der Biezen, E. A., et al. (1997). The Arabidopsis downy mildew resistance gene RPP5 shares similarity to the toll and interleukin-1 receptors with $N$ and L6. Plant Cell 9, 879-894. doi: 10.1105/tpc.9.6.879

Parker, J. E., Szabò, V., Staskawicz, B. J., Lister, C., Dean, C., Daniels, M. J., et al. (1993). Phenotypic characterization and molecular mapping of the Arabidopsis thaliana locus RPP5, determining disease resistance to Peronospora parasitica. Plant J. 4, 821-831. doi: 10.1046/j.1365-313X.1993.04050821.x

Perrone, S., Bui, F., Sutherland, M., and Guest, D. (2000). Gene-for-gene specificity expressed in planta is preserved in cell cultures of Nicotiana tabacum inoculated with zoospores of Phytophthora nicotianae. Physiol. Mol. Plant Pathol. 57, 235-242. doi: 10.1006/pmpp.2000.0300

Rehmany, A. P., Gordon, A., Rose, L. E., Allen, R. L., Armstrong, M. R., Whisson, S. C., et al. (2005). Differential recognition of highly divergent downy mildew avirulence gene alleles by RPP1 resistance genes from two Arabidopsis lines. Plant Cell 17, 1839-1850. doi: 10.1105/tpc.105.031807

Reignault, P., Frost, L. N., Richardson, H., Daniels, M. J., Jones, J. D., and Parker, J. E. (1996). Four Arabidopsis RPP loci controlling resistance to the Noco2 isolate of Peronospora parasitica map to regions known to contain other RPP recognition specificities. Mol. Plant Microbe Interact. 9, 464-473. doi: 10.1094/MPMI-9-0464

Robinson, L. H., and Cahill, D. M. (2003). Ecotypic variation in the response of Arabidopsis thaliana to Phytophthora cinnamomi. Australas. Plant Pathol. 32, 53-64. doi: 10.1071/AP02064

Roetschi, A., Si-Ammour, A., Belbahri, L., Mauch, F., and Mauch-Mani, B. (2001). Characterization of an Arabidopsis-Phytophthora pathosystem: resistance requires a functional PAD2 gene and is independent of salicylic acid, ethylene and jasmonic acid signalling. Plant J. 28, 293-305. doi: 10.1046/j.1365313X.2001.01148.x

Rookes, J. E., Wright, M. L., and Cahill, D. M. (2008). Elucidation of defence responses and signalling pathways induced in Arabidopsis thaliana following challenge with Phytophthora cinnamomi. Physiol. Mol. Plant Pathol. 72, 151161. doi: 10.1016/j.pmpp.2008.08.005

Schlaeppi, K., Abou-Mansour, E., Buchala, A., and Mauch, F. (2010). Disease resistance of Arabidopsis to Phytophthora brassicae is established by the sequential action of indole glucosinolates and camalexin. Plant J. 62, 840-851. doi: 10.1111/j.1365-313X.2010.04197.x

Song, J., Bradeen, J. M., Naess, S. K., Raasch, J. A., Wielgus, S. M., Haberlach, G. T., et al. (2003). Gene RB cloned from Solanum bulbocastanum confers broad spectrum resistance to potato late blight. Proc. Natl. Acad. Sci. U.S.A. 100, 9128-9133. doi: 10.1073/pnas.1533501100

Stassen, J. H. M., and Van den Ackerveken, G. (2011). How do oomycete effectors interfere with plant life? Curr. Opin. Plant Biol. 14, 407-414. doi: 10.1016/j.pbi.2011.05.002

Sun, X., Liu, D., Zhang, X., Li, W., Liu, H., Hong, W., et al. (2013). SLAF-seq: an efficient method of large-scale De novo SNP discovery and genotyping using high-throughput sequencing. PLOS ONE 8:e58700. doi: 10.1371/journal.pone.0058700

Tai, T. H., Dahlbeck, D., Clark, E. T., Gajiwala, P., Pasion, R., Whalen, M. C., et al. (1999). Expression of the Bs2 pepper gene confers resistance to bacterial spot disease in tomato. Proc. Natl. Acad. Sci. U.S.A. 96, 14153-14158. doi: 10.1073/pnas.96.24.14153

Vera Cruz, C. M., Bai, J., Oña, I., Leung, H., Nelson, R. J., Mew, T. W., et al. (2000). Predicting durability of a disease resistance gene based on an assessment of the fitness loss and epidemiological consequences of avirulence gene mutation. Proc. Natl. Acad. Sci. U.S.A. 97, 13500-13505. doi: 10.1073/pnas. 250271997

Wang, G. L., Song, W. Y., Ruan, D. L., Sideris, S., and Ronald, P. C. (1996). The cloned gene, Xa21, confers resistance to multiple Xanthomonas oryzae pv oryzae isolates in transgenic plants. Mol. Plant Microbe Interact. 9, 850-855. doi: 10.1094/MPMI-9-0850

Wang, Y., Bouwmeester, K., Van de Mortel, J. E., Shan, W., and Govers, F. (2013). A novel Arabidopsis-oomycete pathosystem: differential interactions with Phytophthora capsici reveal a role for camalexin, indole glucosinolates and salicylic acid in defence. Plant Cell Environ. 36, 1192-1203. doi: $10.1111 /$ pce. 12052 
Wang, Y., Meng, Y., Zhang, M., Tong, X., Wang, Q., Sun, Y., et al. (2011). Infection of Arabidopsis thaliana by Phytophthora parasitica and identification of variation in host specificity. Mol. Plant Pathol. 12, 187-201. doi: 10.1111/j.13643703.2010.00659.x

Xiao, S., Ellwood, S., Calis, O., Patrick, E., Li, T., Coleman, M., et al. (2001). Broadspectrum mildew resistance in Arabidopsis thaliana mediated by RPW8. Science 291, 118-120. doi: 10.1126/science.291.5501.118

Zhang, Y., Wang, L., Xin, H., Li, D., Ma, C., Ding, X., et al. (2013). Construction of a high-density genetic map for sesame based on large scale marker development by specific length amplified fragment (SLAF) sequencing. BMC Plant Biol. 13:141. doi: 10.1186/1471-2229-13-141
Conflict of Interest Statement: The authors declare that the research was conducted in the absence of any commercial or financial relationships that could be construed as a potential conflict of interest.

Copyright (c) 2015 Meng, Huang, Wang, Wen, Jia, Zhang, Huang, Quan and Shan. This is an open-access article distributed under the terms of the Creative Commons Attribution License (CC BY). The use, distribution or reproduction in other forums is permitted, provided the original author(s) or licensor are credited and that the original publication in this journal is cited, in accordance with accepted academic practice. No use, distribution or reproduction is permitted which does not comply with these terms. 